\title{
Isolated Asymptomatic Pulmonary Artery Sling in a Preterm Neonate
}

\author{
Seda Yilmaz Semerci ${ }^{1}$, Dilek Kurnaz ${ }^{1}$, Tevfik Guzelbey ${ }^{2}$, Seyma Oz ${ }^{3}$ Murat Sahin ${ }^{4}$ and Merih Cetinkaya ${ }^{1}$ \\ ${ }^{1}$ Department of Neonatology, Istanbul Kanuni Sultan Suleyman Training and Research Hospital, Turkey \\ ${ }^{2}$ Department of Radiology, Istanbul Kanuni Sultan Suleyman Training and Research Hospital, Turkey \\ ${ }^{3}$ Department of Pediatrics, Istanbul Kanuni Sultan Suleyman Training and Research Hospital, Turkey \\ ${ }^{4}$ Department of Pediatric Cardiology, Istanbul Kanuni Sultan Suleyman Training and Research Hospital, Turkey
}

\begin{abstract}
Pulmonary artery sling is an uncommon entity in the neonatal period. It is defined as an abnormally originated left pulmonary artery arising from the posterior aspect of right pulmonary artery. Because of the associated structures in this region, mostly originating from sixth aortic arch, tracheobronchial anomalies and congenital heart defects, it frequently accompanies the pulmonary artery sling. Etiology of pulmonary artery sling has not been determined to date. Surgical correction is necessary even for asymptomatic cases because of the high mortality. Postoperative mortality is commonly associated with airway defects. Therefore, prompt diagnosis and timely intervention decrease the morbidity and mortality.

We, herein, present a case of a neonate with isolated pulmonary artery sling un-associated with their developmental anomalies. Antenatal history was positive for maternal hypothyroidism, for which she was taking L-thyroxine. To the best of our knowledge, this is the first pulmonary artery sling case accompanied by maternal hypothyroidism.
\end{abstract}

Key Words: Pulmonary artery sling, Neonate, Hypothyroidism.

How to cite this article: Semerci SY, Kurnaz D, Guzelbey T, Oz S, Sahin M, Cetinkaya M. Isolated Asymptomatic Pulmonary Artery Sling in a Preterm Neonate. J Coll Physicians Surg Pak 2021; 31(03):346-349.

\section{INTRODUCTION}

Pulmonary artery sling is an uncommon entity in the neonatal period. It has been defined as an abnormally originated left pulmonary artery, arising from the posterior aspect of right pulmonary artery. Because of the associated structures in this region, mostly originating from sixth aortic arch, tracheobronchial anomalies and congenital heart defects, it frequently accompanies the pulmonary artery sling. Other system disorders including imperforate anus, biliary atresia, Hirschprung disease, and genitourinary abnormalities, may also be seen either as a part of associations likeVACTERLor syndromes liketrisomy $21 .{ }^{1-3}$

This unusual course of the left pulmonary artery often results in airway obstruction symptoms such as stridor, dyspnea, tachypnea, dysphagia, recurrent or persistent atelectasis, emphysema and frequent lung infections. Although right lung is mostly affected from the anomaly, lower tracheal and left main stem bronchial obstruction may lead to more serious symptoms. The etiology of pulmonary artery sling has not been determined to date.

Correspondence to: Dr. Seda Yilmaz Semerci, Department of Neonatology, Istanbul Kanuni Sultan Suleyman

Training and Research Hospital, Turkey

E-mail: sedayilmazsemerci@gmail.com

Received: June 17, 2019; Revised: November 14, 2019;

Accepted: December 02, 2019

DOI: https://doi.org/10.29271/jcpsp.2021.03.346
The true incidence of pulmonary artery sling is unknown, but increasing numbers of cases have been reported in recentyears by the widespread use of imaging methods. Abnormal pulmonary artery sling runs between the trachea and esophagus, compressing the lower trachea and right main stem bronchus.

Surgical correction is necessary even for asymptomatic cases because of the high mortality. Postoperative mortality mostly depends on associated defects of airway. Therefore, prompt diagnosis and timely intervention can decrease the morbidity and mortality.

Herein, we presenta pretermcase of neonatal isolated asymptomatic pulmonary artery sling accompanied by maternal hypothyroidism; and review the recent literature about this entity. To the best of our knowledge, this is the first pulmonary artery sling case that was possibly related to maternal hypothyroidism.

\section{CASE REPORT}

The infant was the first born of 32 weeks monochorionic diamniotic twin pregnancy as $1300 \mathrm{~g}$ preterm male from a non-consanguineous family. He was hospitalised for prematurity and respiratory distress after Caesarean-section. The presentation of thiscase involved parents' informed consent. Maternal hypothyroidism caused by Hashimoto thyroiditis, and controlled with the use of L-thyroxin and suspected diagnosis of pulmonary vascular sling on fetal echocardiography, was pointed out in 
prenatal history. At the first physical examination, the baby had no pathological sign except tachypnea. Laboratory studies were normal and acute phase reactants were negative. Respiratory distress regressed within four hours and no mechanical ventilation was required. The thyroid stimulating hormone (TSH) level of baby was high as $43 \mathrm{lU} / \mathrm{ml}$ on postnatal day 3, while free thyroxin level was normal. As the infant developed abdominal distention and gastric residues on the second week of life, enteral feeding was stopped and antibiotherapy was started with the diagnosis of necrotising enterocolitis. After five days of total parenteral nutrition, enteral feeding was started and increased meticulously. Cefotaxime, amikacin and metronidazole therapies were stopped after seven days. As the repeated serum TSH level was high $(37 \mathrm{lU} / \mathrm{ml})$, L-thyroxin therapy was started. Pulmonary artery sling was not visualised by the first postnatal echocardiography because of the shunt of ductus arteriosus. Therefore, computerised tomography angiography was performed; and pulmonary artery sling was demonstrated (Figure 1). Following the closeup of ductus arteriosus, pulmonary artery sling could be shown by the second echocardiography performed at postnatal day- 5 (Figures 2 and 3). The second twin was completely normal. As the infant had no pathological signs, corrective surgical operation has been planned to be performed in near future. The infant was discharged on $25^{\text {th }}$ day of life, in a healthy condition, breastfed on demand, and is being followed-up regularly.

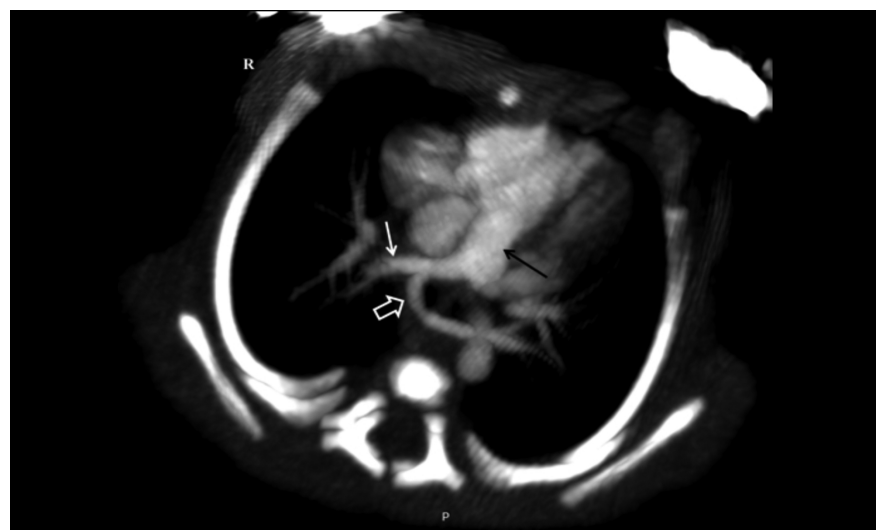

Figure 1: Yellow arrow demonstrates pulmonary artery sling on computerised tomography angiography.

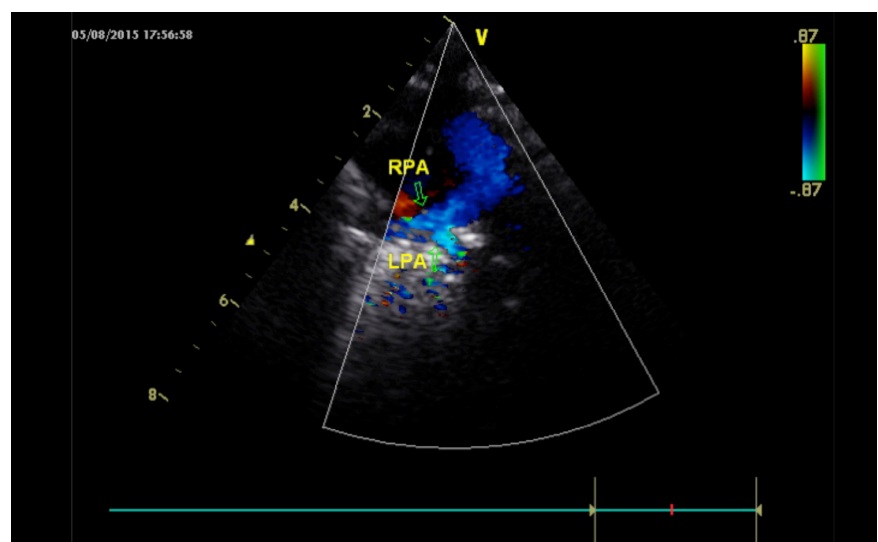

Figure 2: Green arrow points to pulmonary artery sling at DopplerEcho.

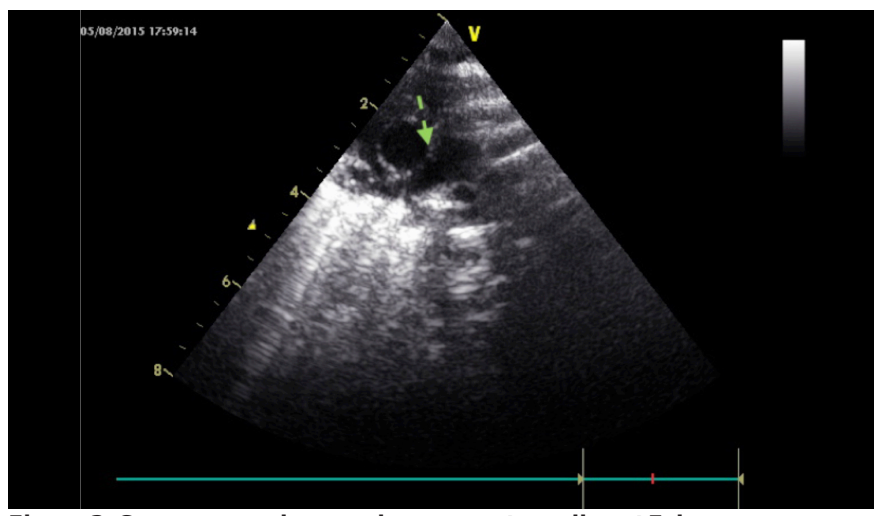

Figure 3: Green arrow shows pulmonary artery sling at Echo.

\section{DISCUSSION}

Pulmonary artery sling is known to be a rare entity, but with the advances of perinatology and imaging methods, an increasing number of pulmonary artery sling cases have been reported in recent years. Although the true incidence of pulmonary artery sling remains unknown, approximately 291 cases have been reported to date. There is no sexual or racial predilection. Twin discordance for the anomaly, similar to our case, is reported only in one article in the literature. ${ }^{4}$

Pulmonary artery sling has been thought to be a developmental failure of sixth aortic arch resulting in an abnormaly originated left pulmonary artery arising from the posterior aspect of right pulmonary artery. Asada et al. hypothesised that pulmonary artery sling might occur when the proximal part of the left sixth aortic arch fails to develop its normal connections to the left lung bud and a 'collateral' compensatory vessel from either the right or the main pulmonary trunk thereby develops. ${ }^{5}$

Considering thyroid gland as the first developing gland of the fetus, its functional or immigration failures may play a role in the development of left pulmonary vascular structures, as in our case. Our patient is the second pulmonary artery sling case reported with neonatal hypothyroidism. The first patient had multiple severe congenital anomalies; whereas, our case had isolated pulmonary artery sling. This may be explained by maternal thyroxin use and normal perinatal thyroxin levels.

Tracheal and/or bronchial stenosis, pulmonary atresia, vascular ring, bridging bronchus, bilateral superior vena cava, and single ventricle were the most reported possibly concurrent abnormalities. ${ }^{6}$

Pulmonary artery sling accompanying Down syndrome is reported only in two cases in the literature. ${ }^{2,3}$ VACTERL association or oculo-auriculo-vertebal spectrum may also occur with pulmonary artery sling. ${ }^{1}$ Isolated pulmonary artery sling, as in our case, is extremely rare in the literature. Furthermore, there are no defined risk factors for the pulmonary artery sling development; maternal hypothyroidism might be a risk factor for pulmonary artery sling in our case. The clinical symptoms of pulmonary artery sling are generally caused by airway obstruction/compression and other coexisting disorders of circulating system. Stridor, dyspnea, tachypnea, dysphagia, recurrent or 
persistent atelectasis, emphysema and frequent lung infections are the initial symptoms that become apparent at different times of life relevant to the severity of the clustering disorders. In severely affected infants, pulmonary artery sling may have a high mortality rate in the first few months of life. Asymptomatic clinical course is considerably rare, as in our case, because of the localisation and/or lack of accompanying abnormalities. Pulmonary artery sling commonly becomes symptomatic in infantile period, but the range is quite wide spanning the first four days of life to adulthood. ${ }^{2}$ Presentation in the neonatal period is rare and mostly related to the other concurrent problems. Therefore, while encountering tracheobronchial or cardiac abnormalities in a neonate, considering sixth arch maldevelopment, possible pulmonary artery sling should be kept in mind. With the advances in perinatology, there are very few reported cases, diagnosed by fetal echocardiography. Our case constitutes one of them..$^{7-9}$ Imaging strategies are helpful for the diagnosis by conventional $x$-ray in the past and recently by echocardiography, MR/CT angiography or direct visualisation during surgery. Echocardiography may not always be successful to demonstrate pulmonary artery sling in the first few days of life. Thus, non-invasive diagnosis of this anomaly with the use of magnetic resonance (MR) or computerised tomography, especially multi-slice CT (MSCT) imaging has beneficial roles in detecting the anomaly by identifying the spatial relationship of the anomalous pulmonary artery to the tracheobronchial structures. It was possible to demonstrate pulmonary artery sling by the MSCT angiography in our case.

The Wells classification is used for preoperative arrangement. The treatment of choice for pulmonary artery sling is surgical correction of the anomaly. Surgical techniques may vary according to the coexisting defects. Postoperative mortality and morbidity have been associated with the accompanying tracheobronchial defects. Surgical treatment is crucial, even for the asymptomatic cases, because of the high mortality of pulmonary artery sling. Intervention may be delayed for asymptomatic infants, as in our case; but if symptoms are apparent, corrective operation should be performed as soon as possible. The early diagnosis and surgical correction usually result in a better prognosis. Therefore, it may be suggested that prompt diagnosis and timely intervention can decrease morbidity and mortality in infants with pulmonary artery sling.

Our patient is of 20 months now and has no symptoms even without surgery. He has been closely followed-up for clinical signs.

In summary, pulmonary artery sling is an uncommon condition and is difficult to diagnose before clinical symptoms occur. Early echocardiography in the first days of life may be inadequate for demonstrating pulmonary artery sling because of ductus arteriosus. Therefore, repeated echocardiography; or angiographic techniques can be more helpful for early diagnosis. Congenital heart diseases, tracheobronchial malformations or genetic disorders can be seen as coexisting conditions. Etiologic factors have not been thoroughly determined yet. To our best of knowl- edge, this is the first pulmonary artery sling case accompanied by maternal hypothyroidism. Further cases are needed to explain the possible role of thyroid hormones on the development of pulmonary artery sling in neonates.

\section{PATIENT'S CONSENT:}

The informed consent is obtained from patient's parents to publish the data concerning this case.

\section{CONFLICT OF INTEREST:}

The authors declared none of the conflict of interest.

\section{AUTHORS' CONTRIBUTION:}

SYS: Contributed to the design of the work, interpretation of data, writing of the first draft of the manuscript revising it critically for important intellectual content and approved the final manuscriptfor publication.

DK: Contributed to the data collection instruments, and also to revising the manuscript, and gave final approval of the version to be published.

TG: Contributed to the design of the work, supervised the execution of the radiologic views and also to revising the manuscript critically, and gave final approval of the version to be published.

SO: Contributed to the conception of the work, drafting the work and revising it critically, and gave final approval of the version to be published.

MS: Contributed to the conception of the work, providing the images, drafting the work and revising it critically for important intellectual content, and gave final approval of the version to be published.

MC: Contributed to the conception of the workand revising itcritically for important intellectual content, and gave final approval of the version to be published.

\section{REFERENCES}

1. Trowitzsch E, Schneider M, Urban A, Asfour B. Congenital pulmonary sling, Aorto-pulmonary window and pulmonary vein obstruction as a diagnostic and therapeutic challenge in an infant with VACTERL association. Clin Res Cardiol 2006; 95(6):338-43. doi: 10.1007/s00392-006-0383-x.

2. Loureiro M, Moreira J, Vaz T, Ribeiro A, Monterroso J, Areias JC. Anomalous origin of the left pulmonary artery (Sling): $A$ case report and review of the literature. Rev Port Cardiol 1998; 17(10):811-5.

3. Takci S, Yigit S, Haliloglu M, Boduroglu K, Kiper N. Pulmonary agenesis and pulmonary sling anomaly in an infant with down syndrome. Genet Couns 2013; 24(3): 337-341.

4. Kaicker J, Kwong J, Dillenburg R, Mondal T. Left pulmonary artery sling: Difference in monochorionic monoamniotic twin presentation. J Neonatal Perinatal Med 2013; 6(3): 273-7. doi: 10.3233/NPM-1367212.

5. Asada D, Oka T, Hamaoka K. Subtype of pulmonary artery sling with ventricular septal defect. Cardiol Young 2011; 21(5):587. doi: 10.1017/S104795111100076X.

6. Tissot C, Darst JR, Kaza AK, Younoszai AK. da Cruz E. Partial left pulmonary artery sling associated with multiple 
ventricular septal defects: A rare congenital anomaly. J Thorac Cardiovasc Surg 2008; 136(4):1085-7. doi: 10.1016/j.jtcvs.2007.12.054.

7. Semple MG, Bricker L, Shaw BN, Pilling DW. Left pulmonary artery sling presenting as unilateral echogenic lung on 20week detailed antenatal ultrasound examination. Pediatr Radiol 2003; 33(8):567-9. doi: 10.1007/s00247-0030942-5.
8. Ishii Y, Miyamoto T, Nakajima K, Tanaka K, Ikeda K, Inamura $\mathrm{N}$, et al. Abnormal cardiac axis as a prenatal marker of left pulmonary artery sling. Pediatr Int 2016; 58(2):158-61. doi: 10.1111/ped.12744.

9. Sezer S, Acar DK, Ekiz A, Kaya B, Bornaun H, Aslan H. Prenatal diagnosis of left pulmonary artery sling and review of literature. Echocardiography 2019; 36(5):1001-1004. doi: 10.1111 /echo.14325. 